\title{
ECONOMÍA Y ARTES: algunas notas críticas sobre sus relaciones
}

\author{
Jorge Xavier Carrillo Grandes
}

Fecha de recepción: 08/2016

Fecha de aceptación: 10/2016

\begin{abstract}
Resumen:
Artes y economía, campos que poco a poco se cruzan teóricamente. Sin embargo, la comprensión que las teorías económicas hacen de las artes prácticamente se ha reducido a contribuciones administrativas del manejo de empresas artísticas y, en forma más general, a dar lineamientos sobre política cultural. Las artes, por su parte, enfrentan las teorías económicas a partir de una noción: mercado del arte. Más allá de estas divergencias, las contribuciones mutuas deben apuntar a ubicarnos histórica, geográfica y epistemológicamente a fin de comprender las relaciones que entretejen las artes, las obras de arte, los artistas, la globalización financiera y las estéticas que se producen desde nuestro Tercer Mundo.
\end{abstract}

Palabras claves: Economía del arte, Mercado del arte, Globalización Financiera, Teorías económicas.

\begin{abstract}
:
Arts and economics are fields that gradually intersect. However, economic theories focus on arts administrative contributions like guidance on cultural policy. Meanwhile, arts face economic theories from the concept of art market. Beyond these conflicts, mutual contributions aim to locate relations that intertwine arts and economy, historically, geographically, and epistemologically to understand Third World's works of art, artists, and aesthetics.
\end{abstract}

Keywords: economics of art, art market, financial globalization, economic theories.

\footnotetext{
Autor:

Jorge Xavier Carrillo Grandes (Quito-Ecuador, 1982). Economista por la Pontificia

Universidad Católica del Ecuador, egresado de la carrera de Teatro de la Universidad Central del Ecuador, tesista de la Maestría en Teatro y Artes Performáticas de la Universidad Nacional de las Artes de Argentina. Artista escénico e investigador independiente.
} 
La economía del arte, como campo de estudio, ha venido conformándose desde hace un poco menos de medio siglo ${ }^{1}$. Con el transcurrir del tiempo, sus investigaciones se han concentrado en aspectos técnicos de la producción y la circulación de las artes -esto es la estructura económica de las artes- pues, si bien el problema del valor en el arte (y de las obras de arte) ha sido analizado con amplitud desde la sociología y la antropología, en el campo de la economía el problema se resuelve de modo directo al igualar precio de mercado con valor, ya que se evalúa la movilización de recursos financieros desde su producción hasta su comercialización. Esta constitución epistemológica da por sentada la noción misma de mercado y permite que la economía del arte devenga en administración de emprendimientos artísticos o en medición de los impactos económico-financieros de apoyos, mecenazgos y subsidios en el área "arte y cultura”, provenientes tanto del sector público como del sector privado ${ }^{2}$ : estructuras de costos, cadenas de producción y de comercialización, marketing

1 El texto considerado fundador del análisis económico del arte es Performing Arts: The Economic Dilemma de William Baumol y William Bowen, publicado en 1966.

2 A partir de la publicación de Baumol y Bowen, se ha admitido, gracias a varias investigaciones que han testeado sus resultados, que las artes, por su estructura, padecen la "enfermedad de los costos" [conocida como Baumol's cost disease y que hoy en día se usa en el análisis de la educación, la salud, la remediación ambiental, etc. Para revisar las variadas aplicaciones de este concepto puede revisarse Baumol (2012)]. Dicha enfermedad consiste en que las artes mantienen un retraso en su productividad debido a que la innovación tecnológica, la cual permite producir más en menos tiempo, no puede modificar las condiciones de producción artística. Vale un ejemplo extremo para clarificar: un cuarteto de Mozart lleva tocar el mismo tiempo hoy que hace 200 años, no así los autos que gracias a esas innovaciones tecnológicas se producen en menos tiempo y con menos mano de obra. Visto de esta manera, el trabajo artístico es el fin mismo, lo relevante, lo que tiene valor (lo que canta el cantante, lo que baila el bailarín, lo que actúa el actor, lo que pinta el pintor, lo que performa el performer, etc.) y no tanto los objetos -registros u obras- producidos que luego pasan a la comercialización de diferentes maneras. cultural, evaluación de los movimientos financieros de las obras de artes y de los artistas en las ferias de arte y en las exhibiciones, análisis econométrico de las subastas, informes sobre la evolución de los mercados por regiones, etc. En ese sentido, la economía del arte se ha nutrido más de los aportes producidos dentro del mismo campo de la teoría económica, como la economía institucional, que de otros campos. De ahí que el valor del arte para la economía pase exclusivamente por el precio pagado por la obra o por la suma de los precios pagados por las copias o (re)presentaciones hechas de una obra. Es más, solo cuando la compra de la obra de arte se concrete, se discutirán los análisis estéticos, sociológicos y antropológicos que revelen información adicional sobre el mundo de las artes, antes no (Hutter \& Shusterman, 2006, pp. 204-5).

Desde el arte, en cambio, la aproximación al concepto "mercado del arte" se lo ha hecho básicamente desde la sociología y la antropología y, como parte de una creencia generalizada, se lo ha equiparado a "comercialización", construyéndolo como figura antagónica de las artes, siendo su antípoda par excellence ${ }^{3}$. Dentro de este ámbito, trabajos como los de Pierre Bourdieu han abierto otras posibilidades de entender el valor no económico

3 En realidad, la comercialización es apenas un aspecto de la economía. En la teoría económica contemporánea, el mercado es entendido como un conglomerado de intercambios de bienes y servicios entre diferentes agentes que deciden demandar y ofertar. Dichos intercambios no solo son comerciales, es decir meros acuerdos de precio. Sucede que, por ejemplo, al no efectuar una transacción se genera un costo de oportunidad (el valor de una opción no concretada); también se ha comprobado que, antes de comerciar o intercambiar, existen una serie de costos previos, llamados 'costos de transacción' que no suelen reflejarse en el precio acordado en el mercado ya que estos están asociados a la información de la que disponen los agentes económicos. Véase Samuelson y Nordhaus (2010), los capítulos 1 y 7. 
del arte en la sociedad, esto es las indagaciones sobre aquello que la economía deja de lado. No obstante, ambas visiones resultan insuficientes, reduccionistas y aislacionistas. ¿Por qué?

Según las teorías económicas convencionales, el mercado posee la capacidad de regular técnicamente todas las actividades económicas; pero, llevado al espacio social más amplio, se ha convertido en un modelo político alternativo, pues incluso las decisiones personales, concebidas como movimientos individuales autónomos y consentidos, acuerdan y encuentran solución a sus posibles conflictos con una aritmética social eficiente, que además resulta correcta social, política y moralmente. Se trata, en definitiva, de un mecanismo eficaz pero sobre todo muy eficiente para solucionar toda clase de problemas... en cualquier nivel. Su proyección histórica desde lo económico se la entiende como un devenir natural tanto de los individuos como de las sociedades locales que confluyen hacia una sociedad mundial: la inevitable aldea global. ${ }^{4}$

Claro queda que existe, en efecto, una amplísima noción denominada "mercado",

4 Rosanvallon (1989) realiza una genealogía de la noción de mercado como noción política. Braudel (1986), por su parte, distingue cómo los distintos niveles de organización económica (desde el autoconsumo hasta la economía de mercado) devinieron, gracias a la participación activa de los Estados europeos, en la expansión de las relaciones sociales capitalistas por el planeta, de ahí que sea entendido como economía-mundo. El concepto par de Wallerstein (sistema-mundo) junto con las teorías de la dependencia, tan en boga durante los últimos cuarenta años del siglo pasado, facilitarían una perspectiva de los procesos de producción y circulación de los bienes y servicios desde nuestras propias realidades e historias artísticas. Se deja de lado, por muy atrayente que sea, este análisis que llevaría a una sospechosa teoría de la dependencia estética, mediada por las finanzas, en el mundo globalizado. Cabe mencionar en este punto el documental The Great Contemporary Art Bubble (Lewis, 2009), para recordar cómo las finanzas globales inflan los precios de las obras y de los artistas, y eso conlleva a una legitimación de ciertas estéticas que son tranzadas en esos mercados. aplicable a todo ámbito, sea desde las teorías económicas como desde las teorías sociológicas y antropológicas, aun cuando sus variopintos momentos sean opacados por lo comercial. Así que hablar del "mercado del arte" tiene implicaciones mayores que sobrepasan los campos ya mencionados y atraviesan lo estético, lo productivo, lo político, lo local, lo regional y lo global.

Pero antes de tentar las relaciones entre esos ámbitos, partamos de la definición sociológica de Andrea Giunta (2008):

El mercado del arte abarca un
circuito de comercialización que
compromete espacios (galerías
de arte) y actores (galeristas y
marchands independientes) que
intervienen en el proceso de
construcción de legitimidades y
de profesionalización del artista.
El mercado fue activo en el
proceso de autonomización del
campo artístico y su existencia
ha condicionado modas, estilos,
formas de producción (p. 3).

La reducción a lo comercial y la intuición de lo productivo y lo político en esta definición no exime una serie de supuestos importantes de la teoría económica ${ }^{5}$ los participantes toman las decisiones para su beneficio, que combinan, en este caso, lo estético con lo económico y que, a su vez, potencia la "autonomización del campo artístico". Las decisiones que toman

5 En este caso, se remite a la teoría neoclásica, la dominante en el campo, en la que los individuos, se supone, toman decisiones racionales tratando de satisfacer de la mejor manera sus preferencias de consumo determinadas con los escasos recursos que cuentan: presupuesto y tiempo. En Samuelson y Nordhaus (2010), capítulo 5, se encuentra una explicación del comportamiento del consumidor y sus preferencias en relación con el tiempo y el presupuesto que dispone, así como una revisión breve de la paradoja del valor y un ejemplo sobre la economía de la adicción. 
los individuos para satisfacer sus experiencias estéticas calzan perfectamente con las disquisiciones de la teoría económica dominante ya que la valoración subjetiva del arte conlleva implícita una reflexión sobre el uso del tiempo y el presupuesto que no sería posible sin esa cierta autonomía económica y social del arte. Mas, reflexionada con detenimiento, esa misma autonomía posibilita estallar la noción mercado del arte, ya que ni el mercado ni el arte son únicos. Todas las artes (escénicas, visuales, sonoras, literarias, performáticas - por poner una división provisoria ) poseen diferentes maneras de circular y comercializar sus obras, pues la propiedad privada, que habilita la entrada en el juego del mercado, se ha extendido en el campo artístico y mina el discurso oficial del acceso universal a las expresiones artísticas. Pero, a su vez, dicha autonomía suelta las amarras del orden artístico e inevitablemente un orden emerge: el mercado de las artes visuales reorganiza el campo y se sofistica tanto que se asume el mercado modelo y subsume el resto de las prácticas económicas de las otras artes gracias al mismo impulso de autonomización ${ }^{6}$. Esta apenas es una parte del

6 Dos ejemplos caben para diferenciar la autonomización en estos tipos de mercado:

A) Los mercados globales de las artes visuales, en los cuales ya se aceptado totalmente el performace-art, son los de mayor desarrollo y de los cuales se genera y publica más información, pues se invierte tanto como en los mercados bursátiles. Véase sitios como Artprice y Artnet en donde se encuentran descritas las metodologías de cálculo de los índices de los mercados de las artes visuales.

B) Las artes escénicas locales, que han entrado en una fase de reconfiguración productiva gracias a la penetración del cine, los "nuevos" medios, el performance-art, los fondos estatales y la creación de ferias artísticas, han debido elaborar nuevas estrategias de financiación en las que las relaciones tipo redes se amplían y permiten una organización social que, puede o no, devenir en organización gremial. De ahí que sea común que un colectivo pase la gorra luego de hacer una presentación en una plaza, luego haga una performance en un teatro, un espacio público o un museo, posteriormente que participe en ferias y en organizaciones gremiales en pro de problema, porque aún queda por investigar cómo ese modelo condiciona al resto de las artes y sus mercados, algo que escapa a estas notas. La idea, más bien, es visibilizar cómo la noción mercado del arte, mediada por las artes visuales, en tanto "bellas artes", subordina las demás artes a las lógicas de la globalización financiera y cómo, a su vez, esto condiciona las estéticas y las poéticas. Metodológicamente, demos un salto necesario para ubicarnos en la actualidad histórica.

Preguntémonos: ¿cabe hablar de los mercados de las artes $\mathrm{y}$, en consecuencia, de cómo se valoran las obras de arte, el trabajo de las y los artistas, y cómo ellas y ellos se integran a las sociedades y el rol que ocupan? Este cuestionamiento, aunque necesario, no ha sido explicitado pues enfoca un problema mayor: no todas las sociedades valoran de la misma manera las obras de arte, ni el trabajo de las y los artistas, así como tampoco aprecian del mismo modo las artes, por más que las teorías económicas, sociológicas y antropológicas del arte marquen la cancha del valor ${ }^{7}$. En el ámbito global, abstracto

la defensa de sus derechos. En este caso, las explicaciones y teorizaciones de Howard Becker (2008) son las que más se acercan a este punto ya que explicita las redes de cooperación del trabajo artístico en el caso expuesto.

En el caso A, las jerarquías de trabajo están definidas y determinadas ex ante; en el caso B, la cooperación surge como manera de sostener la producción estética y económica en complejas redes sociales. Dos tipos de mercados, dos tipos de afrontar la producción artística.

7 Una teoría tan difundida como la pirámide de Maslow, parecería explicar el porqué nuestras sociedades no han desarrollado industrias culturales: la deficiente satisfacción de las necesidades básicas impide arribar a procesos de autorrealización, lo que a su vez redunda en un precario campo artístico. De ahí la justificación para que la política cultural sea asistencialista. Considerada así esta teoría empata a la perfección con la cultura moderna de las artes y una mirada lineal del desarrollo de las sociedades (recordemos la teoría de las etapas del crecimiento económico según W. W. Rostow). Pero, ¿qué acontece en nuestros países en los que las relaciones con las artes resulta más bien sincrética y no tan moderna? 
por demás, las obras de arte y las-los artistas son evaluados con sofisticados procedimientos econométricos, similares a las tasaciones que se efectúan para las empresas en los mercados bursátiles; la diferencia radica en que las empresas son valoradas de acuerdo con sus movimientos de capital en el corto y mediano plazo para continuar innovando, produciendo y atendiendo la demanda, mientras que las obras de arte se evalúan a través de la percepción de dueños de galerías y directores de museo, curadores, críticos e historiadores del arte y la disposición al pago de compradores y coleccionistas, es decir en una abstracción financieramente especializada. No sorprende, pues, que haya empresas dedicadas exclusivamente a realizar pronósticos e informes econométricos sobre su evolución y a evaluar si es rentable o no cierto artista en el corto, mediano y largo plazo $^{8}$. El movimiento de capitales financieros en el nivel global de las artes, no obstante, no puede abstraerse de la lógica de acumulación: invertir ad infinitum en obras y artistas cuyas expectativas creativas son altas de acuerdo con la percepción de los inversionistas que poseen parámetros estéticos muy elásticos. Esta relación finanzas-estética es mucho más complicada pero nos sirve para pensar en la complejidad de los mercados de las artes.

Introduzcamos ahora la variable del "desarrollo geográfico desigual" que por definición es histórica (Harvey, 2003) y permite tomar la globalización desde un ángulo concreto. Al desenhebrar la madeja de dichas construcciones históricas realizadas por personas e instituciones públicas y privadas tanto en países como en regiones y las relaciones que se dan en

8 Para más detalles, tanto históricos como técnicos, puede revisarse el curso online "The Art Market" de Cornell University, dirigido por Cheryl Finley, accesible en: https://confluence.cornell.edu/display/tam2011/Home este sistema-mundo se visibiliza que ciudades, países y regiones juegan roles diferenciados en la producción y la distribución de las obras de arte, y aun cuando estén en consonancia con el acontecer propio de cada lugar, los diferentes mercados de las artes se supeditan de diversas maneras $y$ en relaciones geográficamente desiguales. Por tanto, el valor de las obras de arte y la apreciación de las-los artistas no solo son el resultado de un análisis técnico en el sentido económico-financiero, sino también político, social y perceptivo e intersubjetivo en sentido fenomenológico.

Vayamos ahora a través de la expansión de las redes sociales. Perogrullada denotar que han cambiado las formas de hacer y mostrar obras de arte en diferentes espacios -sean estos virtuales y/o reales que juegan en esa dialéctica entre el acto y el registro (Alonso, 1997)- y han permitido, en elámbito teórico, repensar nociones como estética y poética. En la actualidad apenas se necesita registrarse en alguna de las redes sociales (Facebook, G+, Instagram, etc.) y desde la propia cuenta postear todo lo que se quiera, práctica naturalizada para quienes acceden a internet. Toda una pléyade de artistas de todas las especializaciones difunde sus obras de esta manera. Pareciera que los mercados de las artes son evitables, o al menos que se puede tranzar fuera de ellos. ¿Será esto posible o, más bien, son las estructuras mismas de los mercados de las artes las que están modificándose tan rápido que sus mismos procesos se han inmiscuido en la misma cotidianidad que ya no los percibimos porque se han naturalizado? Imposible no tomar posición frente a los mercados de las artes, ya que mediante aquellos se definen -unas veces más claro y otras no tanto- formas, contenidos y procedimientos artísticos. ${ }^{9}$

9 Piénsese tan solo en Lady Gaga y su relación con artistas 
De nuevo, dudas varias: ¿pueden los artistas rechazar de plano las actividades del "mercado del arte" que atentan contra sus potencialidades artísticas (pues reducen aún más su productividad) y confiar en que otros las hagan? ¿No es esto apelar a que otros se hagan cargo de las actividades del mercado, pues se reconoce -de modo implícito- que son necesarias para hacer circular las obras no tan fáciles de digerir? ¿Qué sucede con los artistas que se hacen cargo por sí mismos de las actividades de mercado y que, sin embargo, producen obras de arte que no siempre responden a los parámetros de esas prácticas artísticas que, si bien alternativas, son hegemónicas? ¿No es esto, en fin, reconocer que hay un sutil pero muy complejo lazo entre estéticas, poéticas y mercado, que los artistas pueden sobrellevar coexistiendo en una estructura subordinada al capital financiero? El caso de Oscar Murillo, el artista colombiano mejor cotizado en la actualidad y con apenas 30 años, es demostrativo: mientras sus detractores lo acusan de ser una ficha que permitió engrosar las ganancias de sus representantes y de las casas de subastas, varios informes económicos rastrean sus actividades, la comercialización de sus obras y las redes financieras en las que se moviliza: cuerpo en tránsito según la apreciación que de su obra hace Legacy Russell, tal como lo hacen los capitales golondrina diríamos nosotros. ${ }^{10}$

$$
\text { Y así se retorna sobre las teorías }
$$

contemporáneos muy cotizados como Jeff Koons y Marina Abramović.

10 Artprice en su informe anual de El mercado del arte contemporáneo en 2014 dedica un epígrafe a su evolución. Halim Badawi, en cambio, hace una crítica más sociológica de este artista en Bussiness is bussiness: especulación y mercado en la obra de Oscar Murillo, reproducido en esferapublica. org. La entrevista a este artista realizada por Legacy Russell se encuentra en bombmagazine.org/article/6921/ económicas. La noción "mercado del arte", lamentablemente, es abstracta y casi espectral: en el campo artístico se la menciona mucho, pero ¿dónde está? Este es uno de los vacíos teóricos más graves, al punto de parecer una mezcla entre point de capiton lacaniano y chivo expiatorio que explica y al que se le puede culpar de toda la debacle y la crisis de supervivencia de las-los artistas aun cuando las artes actuales ${ }^{11}$ sigan más vivan que nunca.

Pero hay que hilar más fino ese revoltijo. Como se mencionó más arriba, ni todas las artes son iguales ni el mercado del arte es unitario, aun cuando la función primordial de este sea lograr la equivalencia de productos y servicios artísticos en todos los niveles. Por más que el performanceart haya cruzado lenguajes artísticos, esto no significó que se haya provocado la conjunción de los mercados de las artes, pues cada arte todavía tiene procedimientos concretos de producción y, por lo mismo, procesos de mercado específicos.

En medio de las diferencias, las instituciones públicasy privadasejecutan políticas culturales que países y regiones elaboran para permitir que quienes se dedican a las diferentes artes puedan vivir: mecenazgos públicos y privados, festivales, centros culturales, redes de trabajo, galerías independientes, ferias de arte, exhibiciones, espacios comunitarios, museos estatales y privados, becas, viajes de intercambio para "actualizar" las prácticas artísticas... esta infinidad de estrategias y lugares dan a entender que, a pesar de las diferencias productivas, las artes no son solo entretenimiento, aun cuando también lo sean. Joost Smiers (2006) no duda en señalar que se trata de un campo conflictivo,

11 Se prefiere emplear "actual" en lugar de "contemporáneo", pues este último se refiere a un tipo de arte y aquel al momento presente en el que diferentes tipos de arte se cruzan. 
pues los intercambios simbólicos producidos desde las artes no son para nada ingenuos, pues siguen supeditados a la lógica económica: "El espacio del arte no es neutral" ya que "después de todo, el arte y los artistas están donde está el poder" (pp. 33-34). Y esa ubicación espacial tiene que ver con la posibilidad de producir transacciones económicas, a pesar de la posición social, política e ideológica de las-los artistas.

Hilando más fino aún, estas contradicciones deben ser revisadas pues, como menciona Marcelo Expósito (2006):

...si he calificado al comienzo como contradictoria la hegemonía simbólica y política de una determinada división del trabajo en el campo artístico, es porque la extensión de un modelo difuso de "artista-gestor" es hoy día tal que la ha desbordado por su base. El trabajo en el campo artístico y cultural responde ahora perfectamente al paradigma del trabajo "comunicativo" que es central en el posfordismo. La hegemonía de determinada división del trabajo artístico es simbólica, por un lado, y se sostiene por intereses económicos e institucionales, por otro. La labor del productor cultural es hoy de facto fundamentalmente comunicativa, lingüística, semiótica, consiste primordialmente en producir mediante el lenguaje procesos que la institución suele instrumentalizar valorizándolos exclusivamente en el momento en que dicha producción se materializa en objetos o acontecimientos económica y políticamente rentables.

Se vuelve menester, pues, desarrollar un análisis transdisciplinario de la producción y circulación de las artes, que no pierda el foco económico. En este cruce van: la teoría económica para revisar el funcionamiento $y$ la interacción de los diferentes niveles de la producción, circulación y consumo de las artes; la sociología y la política para comprender las interacciones sociales y simbólicas; y la estética y la poética para analizar en profundidad las propuestas artísticas en tanto obras. Es decir se deben emplazar las nociones más básicas de las teorías económicas (oferta, demanda, valor) y cruzarlas con las nociones de artes, estética y poética actuales. Una estrategia contaminante en sentido epistemológico.

En definitiva, hace falta comprender cómo el valor, los mercados, las estéticas, las poéticas y el sistema-mundo capitalista se relacionan y se reconstituyen mutuamente, y no solo cómo el mercado condiciona (o determina, dirían los más ortodoxos) estéticas y poéticas en el sistema-mundo geográficamente desigual de la actualidad.

Como ya se mencionó, la economía del arte es casi un administración del arte o más bien un medidor de eficiencia. Las ciencias humanas han abordado el arte desde varias perspectivas y, en la actualidad, algunos autores como Maurizio Lazzarato, Michael Hardt, Antonio Negri, Rebecca Schneider, Isabelle Graw, entre otros, están aproximando nociones de política, estética, poética, mercado y artes, trabajando sobre ellas para entender los cambios que el capitalismo actual ha llevado a cabo para generar un constante flujo de capital al interior de los países, entre ellos y a nivel mundial con las corporaciones.

No obstante, en estas contribuciones importantes hay dos faltantes. Por una parte, ¿cómo se integran los países de América Latina en esta especie de giro esteticista del capitalismo tardío, o sociedad de mercado global? Las 
bienales, como la de San Pablo, contribuyen a medir la incidencia por una parte los artistas latinoamericanos, pero por otra los informes de las empresas cotizadoras del mercado de las artes visuales, señalan a otros artistas latinoamericanos. No sin razón Artprice en su informe (2015) menciona:

El creciente interés por el arte de Latinoamérica se pone de manifiesto en muy distintos aspectos. Las iniciativas museísticas y privadas se han venido multiplicando a lo largo de estos últimos diez años en Europa, Estados Unidos y Oriente Medio. Florecen las iniciativas encaminadas a estrechar lazos y crear oportunidades de diálogo destinadas a constituir la masa crítica y los archivos indispensables para orquestar las adquisiciones más relevantes. [...]

Por tanto, hace ya más de treinta años que el mercado sostiene y difunde el arte latinoamericano a través de las grandes casas de subastas internacionales. Esta especialidad, más discreta que el arte contemporáneo chino o indio, no perdió sin embargo el tren especulativo de 2008, período ávido de los llamados mercados "emergentes" (pp. 41-42).

Y lo corrobora al año siguiente:

En los últimos años, los grandes museos (el Guggenheim en New York, el Centro George Pompidou en París, la Tate Modern en Londres, el MoMA en New York), se han dedicado a recolectar obras latinoamericanas y a recabar información sobre ellas, a veces por medio de fondos de adquisición especializados, como son los fondos latinoamericano y caribeño del MoMA. [...]
En las casas de subastas, el arte latinoamericano ha sido objeto de varias sesiones temáticas en Phillips, Sotheby's y Christie's: subastas sin duda estimulantes para coleccionistas de cierta solvencia que pueden permitirse adquirir valores seguros (Wilfredo Lam, Leonora Carrington o Roberto Malta) a un precio menor que el de las obras de los grandes artistas europeos y estadounidenses de la misma generación, pero también para los aficionados con un poder adquisitivo más reducido, pues muchas obras pueden ser adquiridas por menos de 5000 dólares (las de Nino Cais, Elba Damast, Ivan Capote, Miriam Medrez, Ernesto Pujol o Dr. Lakra, por ejemplo). Se trata de un mercado que está en proceso de consolidación gracias a la intervención de compradores exigentes y prudentes. (Artprice, 2016, pp. 48-49).

Resulta obvio señalar que la especulación financiera, toma al arte latinoamericano como alternativa de fuga y acumulación, en periodos de crisis. En ese sentido, no está en discusión si el valor simbólico del artista merece un análisis exhaustivo, son los precios de mercado los que denotan una trama más compleja: burbujas financieras en su lado más descarnado pero que se muestran como momentos de valorización del arte latinoamericano.

En definitiva, no se trata de "evaluar" qué tan "retrasados" estamos respecto de los países del norte, sino de cómo nuestras artes se integran en los mercados internacionales, a pesar de no participar en ellos directamente. La gestión misma de las artes debe negociar sus conceptos, pues traerlos y aplicarlos en un Tercer Mundo donde las estructuras 
socioeconómicas se caracterizan por ser capitalistas, subdesarrolladas y dependientes, implica posicionarse epistemológicamente $y$ labrar las diferencias teóricas necesarias para nuestras artes, algo que Jameson (1990, p. 116), criticando a Marx y a Adorno, ha sugerido con insistencia para nuestras conceptualizaciones. Las teorías económicas también deben dar el mismo salto. Aún existen discusiones relevantes que deben ser ampliadas para que la economía del arte no se quede en el asunto administrativo y contribuya a explicar relaciones tan complejas como la influencia de las finanzas en la estética y viceversa.

\section{Bibliografía}

Alonso, R. (1997). Performance, fotografía y video: la dialéctica entre el acto y el registro. CAIA. Arte y Recepción.

Artprice. (2015). El mercado del arte contemporáneo en 2014. Obtenido de Artprice: http://imgpublic.artprice.com/pdf/rama2014_ es.pdf

Artprice. (2016). El mercado del arte en 2015. Obtenido de Artprice: http://imgpublic. artprice.com/pdf/rama2016_es.pdf

Baumol, W. (2012). The Cost Disease: Why Computers Get Cheaper and Health Care Don't. New Haven \& London: Yale University Press.

Becker, H. (2008). Los mundos del arte. Buenos Aires-Argentina: Universidad Nacional de Quilmes.

Braudel, F. (1986). La dinámica del capitalismo. México: Fondo de Cultura Económica.
Expósito, M. (octubre de 2006). Entrar y salir de la institución: autovaloración y montaje en el arte contemporáneo. Obtenido de EIPCP: http://eipcp.net/transversal/0407/exposito/es Giunta, A. (2008). Arte, sociología del. En C. Altamirano (Ed.), Términos críticos de la sociología de la cultura. Buenos Aires: Paidós.

Harvey, D. (2003). Espacios de esperanza. Madrid-España: Akal.

Hutter, M., \& Shusterman, R. (2006). Value and the valuation of art in economic and aesthetic theory. En V. Ginsburgh, \& D. Throsby (Edits.), Handbook of the economics of art and culture (págs. 168-208). Oxford-San Diego: North Holland.

Jameson, F. A. (1990). Late Marxism. New York \& London: Verso.

Lewis, B. (Dirección). (2009). The Great Contemporary Art Bubble [Película].

Rosanvallon, P. (1989). Le libéralisme économique. París-Francia: Éditions du Seuil.

Samuelson, P., \& Nordhaus, W. (2010). Economía. México: McGraw-Hill.

Smiers, J. (2006). Un mundo sin copyright. Barcelona-España: Gedisa. 